\title{
Sialic acids in biological and therapeutic processes: opportunities and challenges
}

Article

Accepted Version

Bauer, J. and Osborn, H. M. I. (2015) Sialic acids in biological and therapeutic processes: opportunities and challenges. Future Medicinal Chemistry, 7 (16). pp. 2285-2299. ISSN 1756-8927 doi: https://doi.org/10.4155/fmc.15.135 Available at https://centaur.reading.ac.uk/46099/

It is advisable to refer to the publisher's version if you intend to cite from the work. See Guidance on citing.

To link to this article DOI: http://dx.doi.org/10.4155/fmc.15.135

Publisher: Future Science Group

All outputs in CentAUR are protected by Intellectual Property Rights law, including copyright law. Copyright and IPR is retained by the creators or other copyright holders. Terms and conditions for use of this material are defined in the End User Agreement.

\section{www.reading.ac.uk/centaur}

\section{CentAUR}

Central Archive at the University of Reading

Reading's research outputs online 


\title{
Sialic acids in biological and therapeutic processes - opportunities and challenges
}

\author{
Julia Bauer and Helen M. I. Osborn ${ }^{\dagger}$ \\ ${ }^{\dagger}$ Author for correspondence: \\ Reading School of Pharmacy, University of Reading, Whiteknights, Reading RG6 6AD, UK. \\ Tel.: +44 (0) 1183787338 Fax.: +44 (0) 118378 6562; E-mail: $\underline{\text { h.m.i.osborn@reading.ac.uk }}$
}

\begin{abstract}
It is now well documented that carbohydrates play multiple roles in biological processes, and hence are interesting targets for chemical biology and medicinal chemistry programmes. This review focuses on a subset of carbohydrates, specifically sialic acid containing carbohydrates. It highlights their occurrence and diversity, and presents evidence for their roles in a range of biological pathways. It illustrates that they are targets for novel medicinal chemistry strategies for a range of therapeutic areas, including cancer and immunity. Case studies highlight opportunities and challenges in this area, and sialic acid based drugs that have entered clinical practice, and are promising candidates for future disease intervention schemes, are discussed. The review concludes by highlighting perspectives and emerging roles for these targets.
\end{abstract}

\section{Sialic acids in modern drug discovery: Introduction}

When surveying the literature, it is highly evident that there are many complementary methods for the development of new medicinal chemistry leads, based on a range of therapeutic targets. What has proved particularly exciting is the discovery of novel biological roles for previously known, complex naturally occurring biomolecules, since this opens up a plethora of opportunities for the development of new biological tools to further our understanding of the roles of these biomolecules in natural and disease pathways. The ultimate ambition of such programmes is frequently the identification, and development, of new therapeutic leads and drug candidates. It is in this regard that carbohydrates have proved particularly promising and interesting within chemical biology and medicinal chemistry, as the mammalian glycome possesses an incalculable number of glycan structures, which is produced and regulated by more than 200 enzymes. ${ }^{[1]}$ In mammals, carbohydrates have numerous traditional functions. For example, they are a source of energy and, as constituents of the cell surface, they play structural roles and act as sites of recognition in extracellular communication processes. Many more sophisticated physiological and pathological states have now been attributed to these cell-cell recognition events. For example, fertilisation, cell differentiation, growth and development, as well as the establishment and progression of bacterial 
and viral infections, cancer and immunological diseases, are some wide ranging examples of these important roles. ${ }^{[2-4]}$ Opportunities for translational research within drug development, based on a basic science understanding of the roles of carbohydrates in disease pathways, are being actively pursued, but enthusiasm for such strategies must often be tempered by the high complexity of the structures of the carbohydrates in living systems. This high degree of complexity renders glycan synthesis a particularly challenging task, but this has inspired novel strategies and approaches for their synthesis, including chemical, chemoenzymatic, automated and solid-phase synthesis, as well as innovative array technologies. ${ }^{[5,6]}$

\section{Aims of this review article}

This review focuses on a biologically and therapeutically relevant subset of carbohydrates, specifically sialic acid containing carbohydrates, and highlights their roles in healthy and diseased pathways. Furthermore, it depicts this class of carbohydrates as emerging targets for novel therapeutic strategies, and discusses ongoing challenges and future directions associated with their synthetic and medicinal chemistry.

\section{Introduction to sialic acids}

Sialic acids are based on 5-amino-3,5-dideoxy-D-glycero-D-galacto-nonulosonic acid (1) (Figure 1) and have the following principal structure.

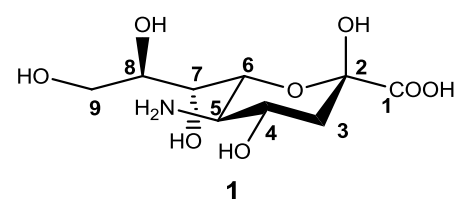

Figure 1. Principal structure of sialic acids.

They are widely distributed in Nature, mainly in vertebrates, but also in higher invertebrates and some types of bacteria. ${ }^{[7,8]}$ They are found in all cell types at the terminal position of cell surface glycoconjugates and as such function as primary recognition sites for carbohydrate binding proteins allowing them to play crucial roles in cell communication processes. ${ }^{[8]}$ At physiological $\mathrm{pH}$, they are deprotonated. ${ }^{[9]}$

Due to their terminal position and abundance in glycoconjugates, sialic acids have been extensively studied in order to elucidate their biological roles as cell surface components as well as recognition elements in cell communication processes. ${ }^{[10]}$ Synthetic efforts span from the preparation of small molecules, i.e. manipulation of sialic acid itself, to the synthesis of complex natural and unnatural sialosides obtained by both chemical and enzymatic methods. From a chemical perspective, sialic acids differ from other carbohydrates in a number of ways and hence present a number of synthetic 
challenges: (1) Sialic acids are acidic sugars, which consist of a 9-carbon backbone; they carry a carboxylic acid functionality at position 1 , which requires protection by esterification in the majority of synthetic schemes, (2) Sialic acids do not possess an anomeric proton, thus traditional ${ }^{1} \mathrm{H}$ and ${ }^{13} \mathrm{C}$ NMR spectroscopic methods for the assignment of the stereochemistry of newly formed glycosidic centres (based on coupling constants) cannot be utilised, (3) Sialic acids belong to the class of keto sugars and are often prone to elimination reactions. Unlike aldoses, such as e.g. glucose, they appear as ketoses in their ring-opened form, (4) An additional feature of sialic acids is the glycerol side chain (positions C 7, C 8 and C 9) providing vicinal diol moieties, which are relatively flexible and unhindered ${ }^{[11]}$, and (5) In Neu5Ac (2), the $N$-acetamido functionality has electron-withdrawing properties. In line with the majority of chemical synthesis strategies for carbohydrates, the hydroxyl groups in sialic acids require protection, in order to achieve regiospecific glycosidation reactions, and selective protection of specific hydroxyl groups in sialic acid often requires advanced protecting group strategies. Moreover, protection of hydroxyls as acetate esters bears the risk of protecting group migration, often resulting in a mixture of compounds.

\section{Diversity, occurrence and functions of sialic acids}

\section{Diversity}

The family of sialic acids is characterised by a high structural and functional diversity. In Nature, more than 50 structurally different sialic acid derivatives exist. ${ }^{[12]}$ The diversity arises from the type of glycosidic linkage through which sialic acid moieties are attached to other glycan units, and from sialic acid specific natural modifications. The two most abundant members of the sialic acid family are Neu5Ac ( $N$-acetylneuraminic acid (2)) and Neu5Gc ( $N$-glycolylneuraminic acid (3)), followed by KDN (2-keto-3-deoxy-nononic acid (4)) and Neu (neuraminic acid (1)). ${ }^{[7]}$

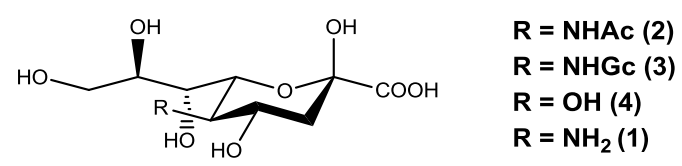

Figure 2. Structures of the most abundant members of the sialic acid family.

These sialic acids exist in $\alpha 2,3$ - and in $\alpha 2,6$-linkage to galactose-containing glycans or in $\alpha 2,6$-linkage to $\mathrm{N}$-acetylgalactosamine residues (Figure 3). They can also be linked via an $\alpha 2,8$-linkage to other sialic acid moieties (polysialic acid). ${ }^{[7]}$ 

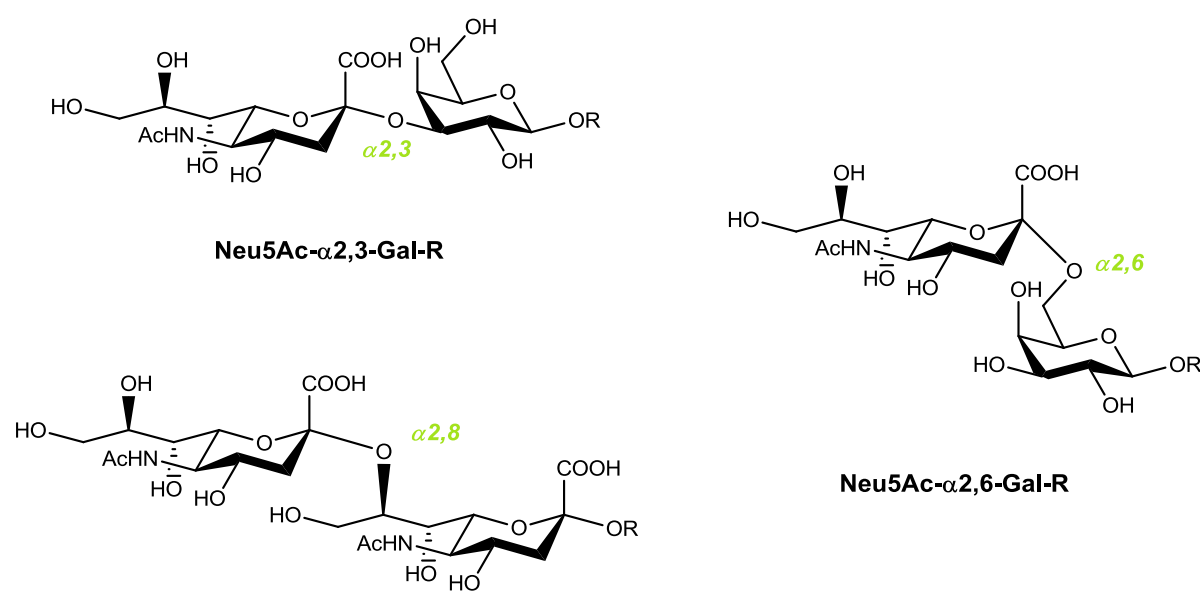

Neu5Ac- $\alpha 2,8-$ Neu 5 Ac-R

Figure 3. Different linkage types of sialic acids to underlying carbohydrate units.

Additional diversification results from modifications of the four above mentioned examples of sialic acid core structures by $\mathrm{O}$-acetylation, $\mathrm{O}$-methylation or by the introduction of $\mathrm{O}$-lactyl groups, sulfate or phosphate esters at positions $4,7,8$ and/or 9 . These derivatives are generated by enzymatic catalysis. For example, $O$-acetyltransferases catalyse the synthesis of $O$-acetylated sialic acid derivatives. The mechanisms for the generation of other modifications e.g. lactamisation, lactonisation or de-N-acetylation are unknown. ${ }^{[8]}$ It is noteworthy that Neu5Gc (3) does not occur physiologically in humans, but is found in animal tissue. The reason for this is the lack of the enzyme CMP-Neu5Ac hydroxylase, which is responsible for the synthesis of CMP-Neu5Gc from CMP-Neu5Ac, in humans. ${ }^{[8,9]}$

\section{Occurrence of sialic acids}

The occurrence of sialic acids in mammalian systems has been extensively studied and is extremely broad and tissue/species specific. For example, they are found as components of milk oligosaccharides and also occur as part of glycoproteins in blood, sera or plasma of mammals. Human sera are mainly composed of Neu5Ac (2) and Neu5Ac9Lt (Lt = lactoyl) (5) as well as small amounts of $O$-acetylated sialic acid derivatives, such as $\mathrm{Neu}, 9 \mathrm{Ac}_{2}(6)$. The ratios and composition of sialic acid derivatives in serum glycoproteins differ substantially amongst mammalian species with respect to the level and position of $O$-acetylation as well as the presence of Neu5Gc (3) ${ }^{\left[{ }^{[9]}\right.}$ Sialic acids also occur on epithelial and mucous glycoproteins. For example, early studies of the composition of human colonic mucous glycoproteins revealed the presence of Neu5Ac as well as acetylated derivatives thereof (Neu5,9Ac 2 (6) and Neu5,7,9 $\mathrm{Ac}_{3}$ (7)) (Figure 4). ${ }^{[9]}$ Not only glycoproteins, but also glycolipids, in particular gangliosides, are terminated by sialic acids. ${ }^{[8,9]}$ 


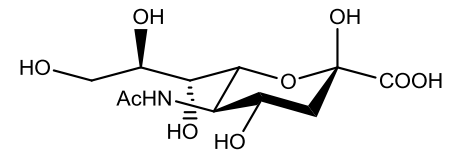

2

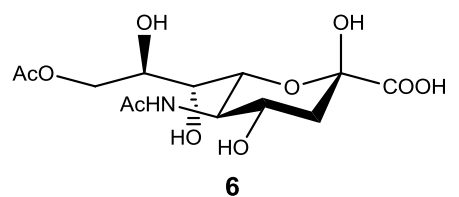

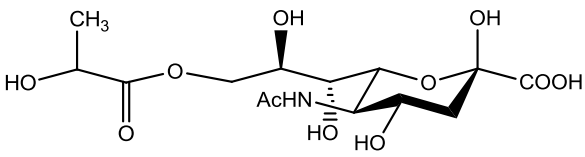

5

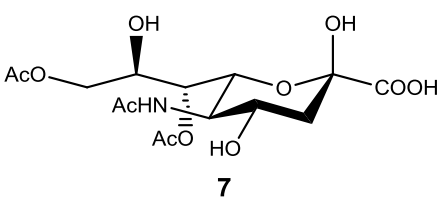

Figure 4. Naturally occurring sialic acid derivatives.

\section{Functions of sialic acids}

The wide distribution and abundance of sialic acids as components of glycoconjugates supports their broad biological significance. Sialic acids influence the structure and function of glycoconjugates and, as ligands for lectins, antibodies and enzymes, they mediate essential cell interaction processes in both healthy and diseased states and as such play an important role in cell communication and information transfer. ${ }^{[1,8,10]}$

The structural and physical functions of sialic acids comprise e.g. stabilisation of erythrocytes and prevention of aggregation of blood components by their negative charge and hydrophilicity. As constituents of glycoproteins (e.g. hormones and enzymes), sialic acids also affect their stability and functionality. A repertoire of biological effects can be derived from sialic acid containing glycans functioning as ligands for glycan binding proteins, such as animal lectins (e.g. selectins and siglecs), viral lectins (hemagglutinins) and bacterial lectins (adhesins and toxins). Most extensively documented are the interactions of sialic acids with siglecs and selectins. The interactions of sialosides with lectins from the siglec family are crucial in the regulation of the immune response, immune cell functions, cell growth and survival. ${ }^{[10,13]}$ They also play a pivotal role in the central and peripheral nervous system by controlling and stabilising components of neuronal cells. ${ }^{[9]}$ Cell adhesion processes are mediated by the interactions of sialylated glycoconjugates (Lewis antigens) with selectins, which effect the homing of lymphocytes, the recruitment and direction of leukocytes to sites of inflammation. This type of interaction is also involved in angiogenesis, metastasis, thrombosis and cancer. ${ }^{[1]}$ In addition, sialic acids represent target structures for viral lectins, such as the hemagglutinin of influenza viruses. The interaction of this viral protein with sialosides on the host cell promotes the establishment of the infection by binding to and entering the host cell. ${ }^{[14]}$ The adhesion of bacterial lectins, e.g. Helicobacter pylori and Mycobacterium tuberculosis adhesins or Cholera and Tetanus toxins, to host cells via interaction with their sialic acid containing ligands are involved in the infection process with these pathogens. ${ }^{[15]}$ Sialic acids have also been found to be exploited by microbial pathogens to mask their antigenic sites. For example, the parasite 
Trypanosoma cruzi is able to transfer host cell sialic acid molecules onto its own glycan units in order to prevent recognition and elimination by host immune cells. ${ }^{[15]}$ Sialic acids are also involved in reproductive and developmental processes and sialylation of hormones, such as the follicle stimulating hormone (FSH) and human chorionic gonadotropin (hCG), contributes to their stability and functionality. ${ }^{[15]}$

The following sections provide a more in-depth discussion of the roles of sialic acid containing carbohydrates in specific biological pathways, to highlight the opportunities and challenges for the development of new therapeutic agents based on carbohydrates.

\section{Sialylation in cancer and immunity}

Sialic acids are found at the terminal position of glycans of all cell types. However, in diseased states, such as cancer or immunological disorders, the sialylation profile of cells in affected tissues is altered. This alteration in cell surface sialylation can be caused by under-, over- or neoexpression of certain glycan structures. ${ }^{[3]}$ Changes arise from modification of glycan core structures, e.g. by sialylation of additional oligosaccharide branches of $\mathrm{N}$-glycans ( $\beta 1,6-$ GlcNAc branching) or from manipulation of terminal glycan sequences, such as premature sialylation of truncated saccharide units. Ultimately, these aberrant cell glycosylation profiles are a result of changes in the expression level of enzymes. ${ }^{[3]}$

For example, the downregulation of sialyltransferases, which are involved in glycosylation processes of immune cells, e.g. ST3Gal-I and ST6Gal-I, lead to a lack of cytotoxic T-cells and attenuation of B-cell signaling, results in a severe impairment of immune mechanisms. ${ }^{[16]}$ In rheumatoid arthritis, an incomplete glycosylation of IgG with galactose and sialic acid moieties has been found to contribute to the development of this condition. ${ }^{[1]}$

In contrast, in cancer, the expression of sialyltransferases is often upregulated. For example, ST3Gal-I is upregulated in human breast cancer[16] and ST6Gal-I is upregulated in human breast and colon cancer, which results in altered cell sialylation patterns. This affects the interactions of the tumour cell with other cells with respect to cell adhesion, migration and their tendency to metastasise. ${ }^{[4]}$

In a recent study on the role of natural killer cells in cancer immunity, it was revealed that cell surface sialylation correlates with natural killer (NK) cell cytotoxicity. Hypersialylation is exploited by the tumour cell to evade recognition by NK cells, thus protecting the tumour from an attack by the immune system. To probe this, a number of synthetic sialic acid containing glycopolymers including Neu5Ac (2), $\alpha 2,3-N e u 5 A c-L a c N A c$ (8), $\alpha 2,6-N e u 5 A c-L a c N A c$ (9) as well as sLe ${ }^{x}$ (10) and GD3 (11) (Figure 5) residues were internalized into cancer cell membranes by glycocalyx engingeering and their potential to inhibit NK cell killing was assessed. It was confirmed that the glycopolymers bearing 
Neu5Ac (2) alone were the most effective determinants in decreasing NK cell activity. It was established that the lectin, Siglec-7, which is ubiquitously expressed on human natural killer cell, functions as the mediating receptor in this context. ${ }^{[11]}$

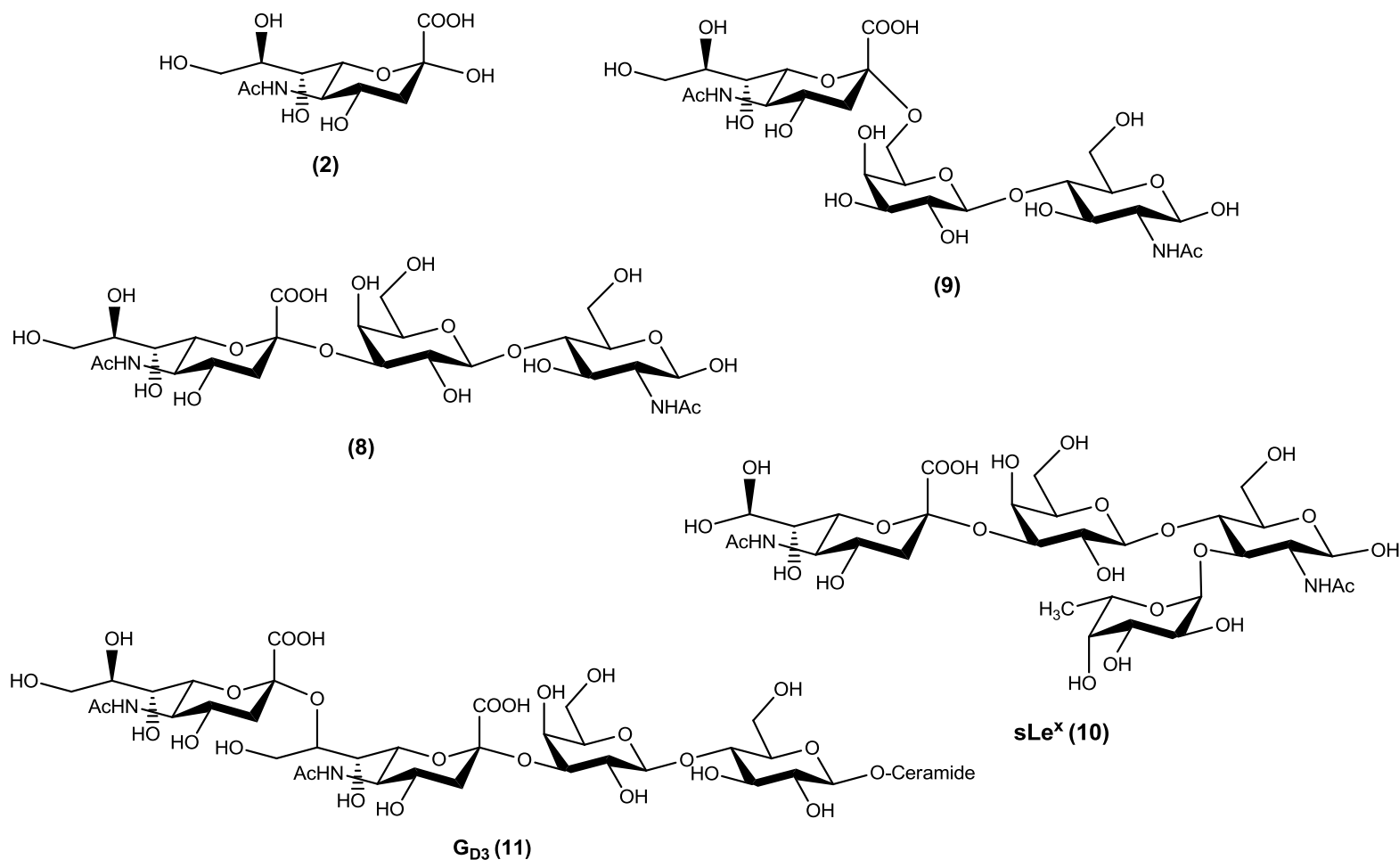

Figure 5. Sialiosides that were incorporated into synthetic glycopolymers to study the role of natural killer cells in cancer immunity.

In addition, it was found that hypersialylation not only protects the cancer cell from NK cell killing, but may also form therapeutically induced antibody-dependent cell cytotoxicity (ADCC), thus interfering into reactions of both the innate and adaptive immune response, providing an advantage for tumour cell. These findings pave the way for the development of novel therapeutic agents that target the Siglec-7 mediated mechanism of NK cell immunoevasion. They may also be of use to further examine the dependence of the cell sialylation pattern on the efficacy of ADCC therapy. ${ }^{[1]}$

Another study that investigated the potential of Siglec-7 as a target for the therapeutic intervention of cancer was presented by Rillahan et al., who identified a selective high-affinity ligand for Siglec-7 using a microarray approach. On-chip derivatisation of sialic acid containing glycans allowed generation of a sialoside library of more than 1000 sialic acid analogues that were subsequently probed with Siglecs-7, -9 and -E. The structural diversity of ligands to be tested, resulted from the reaction of 10-15 alkyne-functionalised sialosides with a range of azide substituents through $\mathrm{Cu}(\mathrm{I})$ catalysed azide-alkyne cycloaddition (CUAAC). Screening of the arrays with Siglecs showed promising results and revealed a 5-azido-fluorescein substituted sialoside analogue (12) as a high-affinity ligand for Siglec-7 (Figure 6). In order to probe the interactions of the newly identified sialoside ligand with 
its receptor on cells, the sialoside analogue was incorporated into a liposomal nanoparticle formulation, which was then subjected to binding studies with Siglec-7 expressing cells. Indeed, the modified sialoside derivative bound selectively and with high affinity to Siglec-7. Its affinity even exceeded that of a sialoside derivative with an azido-adamantane substituent (13) (Figure 6), which had previously been reported for its high affinity to Siglec-7. Further evidence of the binding event was provided by investigating the crystal structure of the lectin in complex with the sialoside analogue. ${ }^{[17]}$
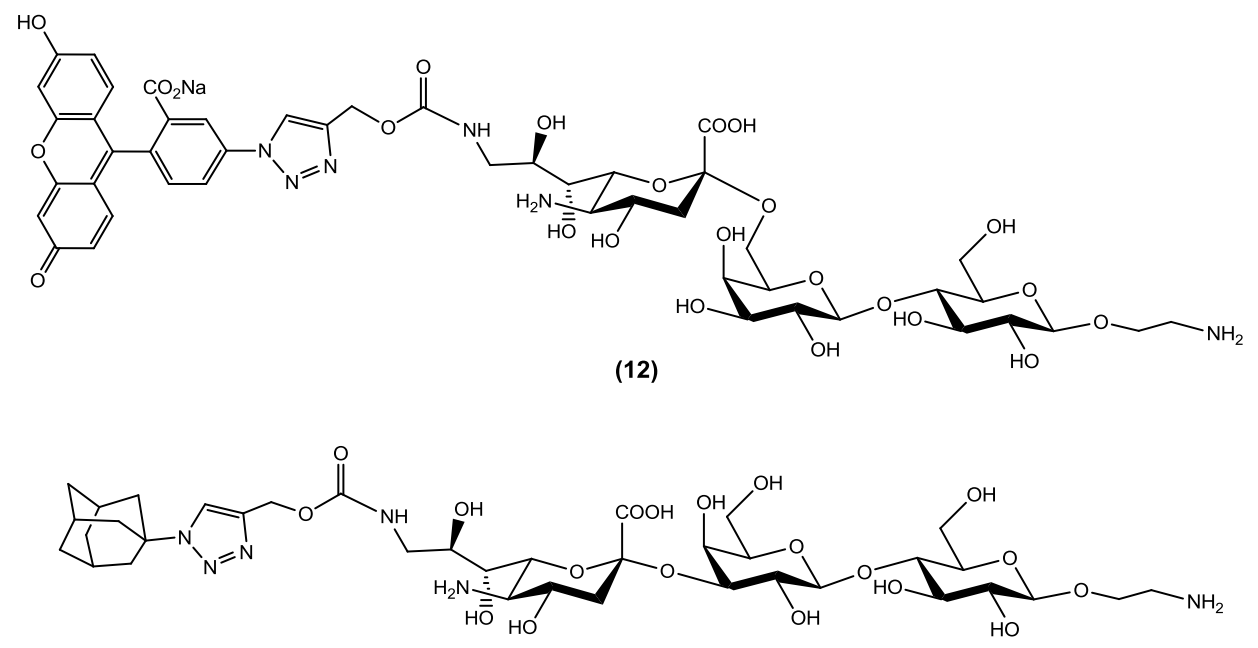

(13)

Figure 6. High-affinity ligands for Siglec-7.

The identification and characterisation of this sialic acid ligand-protein interaction is the foundation for the design and development of innovative therapeutics, which contain sialoside components that specifically target siglecs. These findings add new perspectives to anticancer therapies and are valuable for the elucidation of Siglec utility and function. ${ }^{[17]}$ In addition, nanoparticle formulations proved to be viable approaches for the delivery of potential therapeutic agents to the target cell. Further advances in this area have been reviewed by Macauley et al.. ${ }^{[18]}$

\section{Tumour-Associated Carbohydrate Antigens (TACA)}

The upregulation of enzymes, e.g. sialyltransferases and fucosyltransferases, and the presence of their glycosylation products, i.e. tumour-associated carbohydrate antigens (TACA), is associated with malignant forms of tumours, metastasis formation and a poor prognosis. ${ }^{[4,19]}$ Typical TACA are sialyl Lewis antigens (sLe $(\mathbf{1 0})$, sLe $\left.{ }^{a}(\mathbf{1 4})\right)$ (Figure 7), the sialyl Thomsen nouvelle antigen (sTn) (15) and subsets of gangliosides. Whereas $s \mathrm{~L}^{\mathrm{x}}(\mathbf{1 0})$ and $s \mathrm{Se}^{\mathrm{a}}(\mathbf{1 4})$ antigens are only found at very low levels in healthy tissue, their expression is significantly increased in carcinoma cells. This is due to the upregulation of the enzymes ST3Gal-III and FucT-III, which are responsible for the synthesis of sLe (12) and ST3Gal-IV, ST3Gal-VI and FucTVII, which catalyse the synthesis of SLe ${ }^{x}(\mathbf{1 0}) .{ }^{[19]}$ 


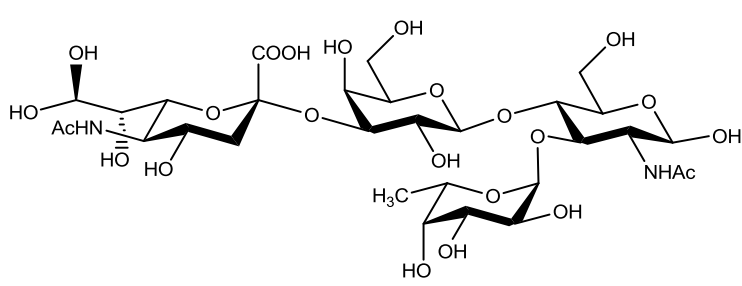

$s L e^{x}(10)$

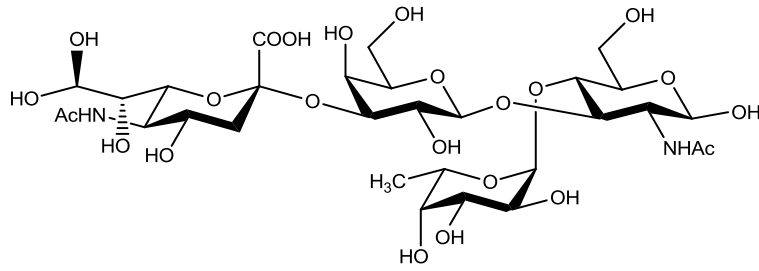

sLe $e^{a}(14)$

Figure 7. Structures of sialyl Lewis antigens $s \mathrm{~L}^{\mathrm{x}}(\mathbf{1 0})$ and $s L \mathrm{e}^{\mathrm{a}}(\mathbf{1 4})$.

In a study to investigate the metastatic potential of sLe antigen-expressing tumour cells, it was shown that a downregulation of sialyltransferases and fucosyltransferases resulted in a reduced cell surface sialylation and in a reduction of the levels of TACA. As a consequence, the cell's adhesion and migration potency decreased and its metastatic activity was minimised. ${ }^{[20]}$

Another aberrant carbohydrate epitope that is rarely found in healthy tissues is the sTn antigen (15). The sTn antigen (15) occurs on epithelial cancer cells and is present on $25-30 \%$ of all breast tumours. It is generated by premature sialylation of its precursor Tn antigen (16) with ST6GaINAc I. ${ }^{[20]}$ The overproduction of gangliosides in cancer represents an additional example of an altered expression profile of glycoconjugates under physiological conditions. The central nervous system (CNS)-specific disialylated glycolipid $G_{D 3}(\mathbf{1 1})$ was also detected in tumour tissues, with 9-O-acetylated sialic acid derivative-terminated glycoforms being overexpressed in a particular type of breast cancer (Figure 8). ${ }^{[19-21]}$

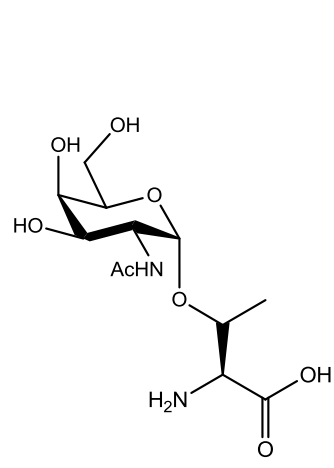

Tn-antigen (16)

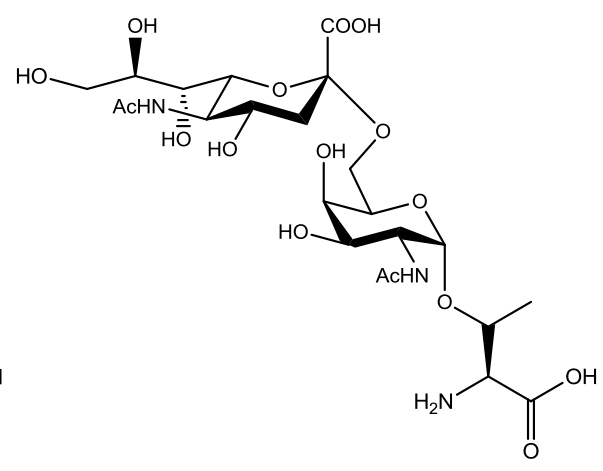

sTn-antigen (15)

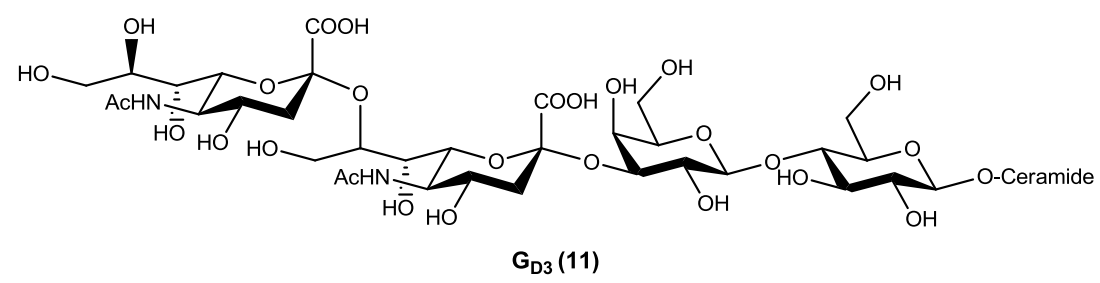

Figure 7. Structures of the tumour-associated carbohydrate antigens Tn-antigen (16), sTn-antigen (15) and $\mathrm{G}_{\mathrm{D} 3}(\mathbf{1 1})$. 
The examples given above demonstrate some of the roles of sialic acids and sialylation in cancer and immunity and highlight key roles of sialic acids in pathological states. Although the entirety of processes, in which sialic acids are involved are not completely understood and discovered, the existing literature indicates their importance in a variety of physiological and pathological conditions and their potential to be used as promising targets for disease intervention.

\section{Sialic acids in drug discovery: Opportunities and challenges}

Multiple conventional medicinal chemistry opportunities exist for the development of drug leads, based on carbohydrates. For example, inhibitors for the enzymes responsible for preparing the disease-associated carbohydrates can be designed, synthesized, and analysed. Inhibitors for the carbohydrate-binding receptors involved in the disease pathways can also be pursued. However, carbohydrate-derived drug candidates present challenges, particularly with respect to their pharmacokinetic profiles. Their bioavailability is poor, since, by nature, carbohydrates are highly polar, which makes passive absorption impossible. Active transport systems are required to facilitate the passage through membranes. Further, carbohydrate-based drugs typically have a short plasma half-life, a poor metabolic stability and are rapidly excreted. The sections below provide case studies to exemplify medicinal chemistry approaches to identify useful sialic acid containing compounds for drug discovery, as well as tools that have been developed to optimise their pharmacokinetic profiles. $^{[21]}$

\section{Design and development of chemical inhibitors}

The elucidation of the structure of mammalian sialyltransferases complexed with their ligands by Xray crystallographic analysis allows a better understanding of substrate specificities and facilitates the design of inhibitors. ${ }^{[16]}$ Inhibition of sialyltransferases could effectively modulate cell surface sialylation and the generation of cancer-specific sialylated epitopes. ${ }^{[19]}$ The expression of glycosyltransferases, which is regulated by intracellular signalling pathways and transcription factors, is directly linked to the synthesis of functional glycans. ${ }^{[2]}$ For example, the absence of ST3Gal-IV and ST3Gal-VI leads to a deficiency in sialic acid containing ligands for selectin interactions, thus influencing selectin-mediated processes, such as leukocyte homing and leukocyte recruitment to sites of inflammation. ${ }^{[22]}$

Sialoside-selectin interactions cannot only be prevented by the inhibition of ligand synthesis, but also by blocking lectin binding by selectin-antagonists. A number of selectin antagonists (Figure 9), e.g. bimosiamose (TBC-1269) (17) and GSC-150 (18) were developed for the treatment of inflammatory disorders, autoimmune diseases and metastatic cancers in order to reduce leukocyte trafficking to inflamed tissues and to impede the dissemination of tumour cells. ${ }^{[1,21]}$ Ernst and co-workers designed 
a sLex-derived inhibitor of E-selectin, CGP69669A (19), which revealed a 12-fold higher affinity compared to the natural ligand $s \mathrm{e}^{\mathrm{x}}$ by replacing the Neu5Ac moiety of $s \mathrm{~L}^{\mathrm{x}}$ with $(S)$-cyclohexyl lactate. Kunz and co-workers extended this approach by synthesising modified sLe -containing $^{\mathrm{x}}$ glycopeptide analogues for the inhibition of E-selectin. ${ }^{[23]}$ A novel P-selectin antagonist, GMI-1070 (20), developed by Ernst and associates ${ }^{[21]}$, is currently being investigated in Phase II clinical trials for the treatment of an acute complication, sickle cell crisis, associated with sickle cell disease (SCD). ${ }^{[24,}$ ${ }^{25]}$ Bimosiamose (TBC-1269) (17), a P-selectin antagonist, successfully completed Phase II clinical trials for the indications psoriasis and chronic obstructive pulmonary disease (COPD). ${ }^{[25,26]}$ Detailed biological data for the compounds shown below were not accessible from the literature.
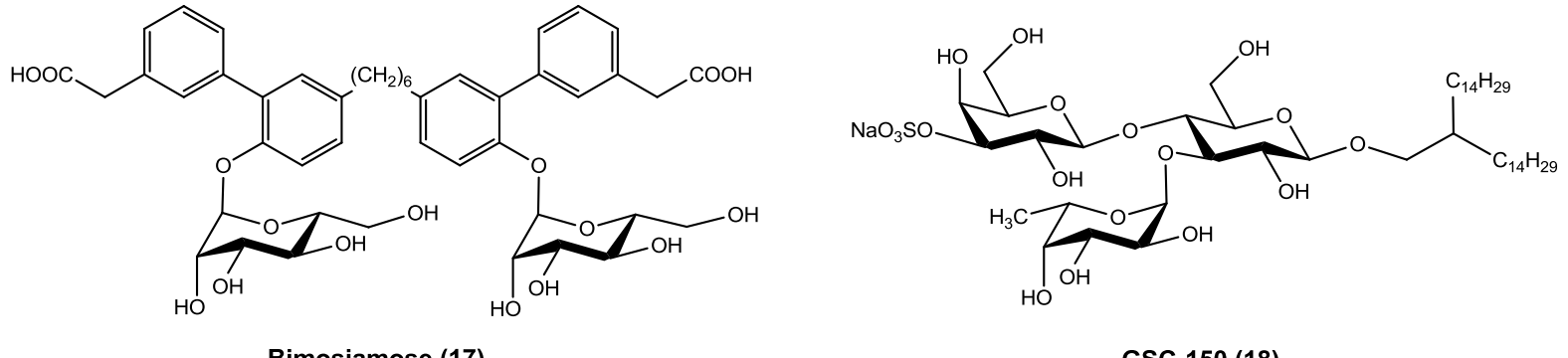

GSC-150 (18)
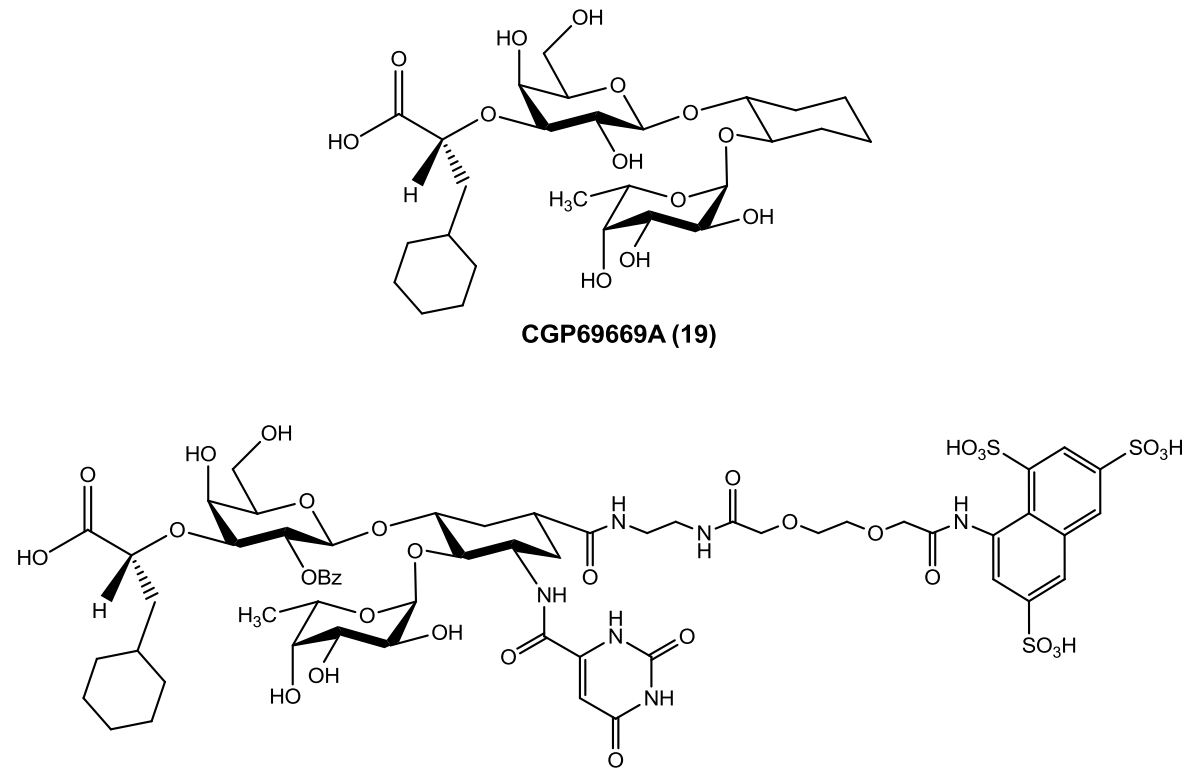

GMI-1070 (20)

Figure 9. Structures of a selection of selectin antagonists.

Other sialic acid-based, small molecular weight antagonists, which target the interactions of sialosides with the lectin MAG, were developed by Mesch et al. as a therapeutic strategy to promote axon regeneration after neuronal injury. ${ }^{[27]}$

Zanamivir (21) (Relenza) and oseltamivir (22) (Tamiflu) (Figure 10) represent two examples of sialic acid based inhibitors of the viral enzyme neuraminidase, which is involved in the establishment of 
influenza infections. ${ }^{[21]}$ As oxocarbenium intermediate analogues, the two drugs mimic the transition state of bound sialic acid residues in the neuraminidase's active site, hence inhibiting sialic acid cleavage from the host's cell surface as well as the release of newly replicated viruses. ${ }^{[28]}$

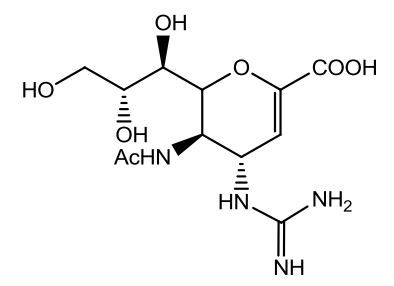

Zanamivir (21)

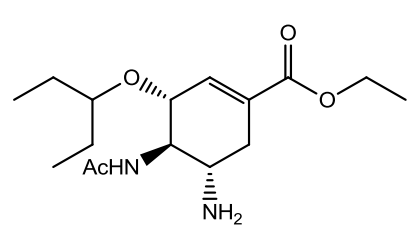

Oseltamivir (22)

Figure 10. Structures of the neuraminidase inhibitors zanamivir (21) and oseltamivir (22).

\section{Overcoming pharmacokinetic limitations}

In order to improve their pharmacokinetic characteristics, potential lead candidates need to systematically undergo structural modification processes, such as e.g. the replacement of certain groups by bioisosteric functionalities, the introduction of hydrophobic moieties and/or the incorporation of a prodrug strategy. ${ }^{[21]}$ The resulting, structurally modified compounds mimic the biological activity of their carbohydrate precursors and are also termed 'glycomimetics'. ${ }^{[21]}$ The prime examples of such glycomimetic drugs are the above mentioned neuraminidase inhibitors zanamivir (21) and oseltamivir (22).

An additional aspect that needs to be considered in the design of drugs that target glycan-lectin interactions is the low affinity and multivalent nature of the binding events between lectins and their ligands. This hurdle can be overcome by the synthetic introduction of functionalities that increase the affinity though favourable interactions within the binding site or by the multivalent presentation of ligands to achieve sufficient avidity. ${ }^{[21,29]}$

The abundance of glycan structures within an organism and their significance in the regulation of vital functions highlights the crucial problem of selectivity. The inhibition of an enzyme that is involved in the synthesis of tumour-associated glycans would also affect the functionality of enzymes at remote sites, which catalyse physiologically relevant glycan synthesis. Further, the interference in lectin-ligand interactions, e.g. the interactions between sialic acids and selectins, can be advantageous for the attenuation of inflammatory disorders or the decrease of the metastatic potential of tumours, but blocking of selectin interactions may also impair essential physiological processes and thus lead to severe side-effects. Thus, targeting drug delivery approaches are required to overcome the problem of selectivity. 


\section{Targeted drug delivery approaches}

The issue of selectivity has been addressed by Paulson and co-workers, who developed a liposomal nanoparticle strategy to target B cell lymphoma. ${ }^{[30]}$ For this approach, the chemotherapeutic doxorubicin was incorporated into liposomes, similar to the liposomal nanoparticle formulation Doxil. Selectivity for B cells was achieved by decorating the liposomes with the high-affinity ligand, 9$N$-biphenylcarboxyl (BPC)-Neu5Ac- $\alpha 2,6-G a l-\beta 1,4-G I c N A c$ (23), which is specifically recognised by the lectin CD22 (Siglec-2), which is exclusively expressed on B cells. From a synthetic point of view, the trisaccharide derivative BPC-Neu5Ac- $\alpha 2,6-G a l-\beta 1,4-G I c N A c$ (23) was conjugated to a commercially available pegylated lipid, which was then subjected to vesicle formation under doxorubicin encapsulation (Figure 11).

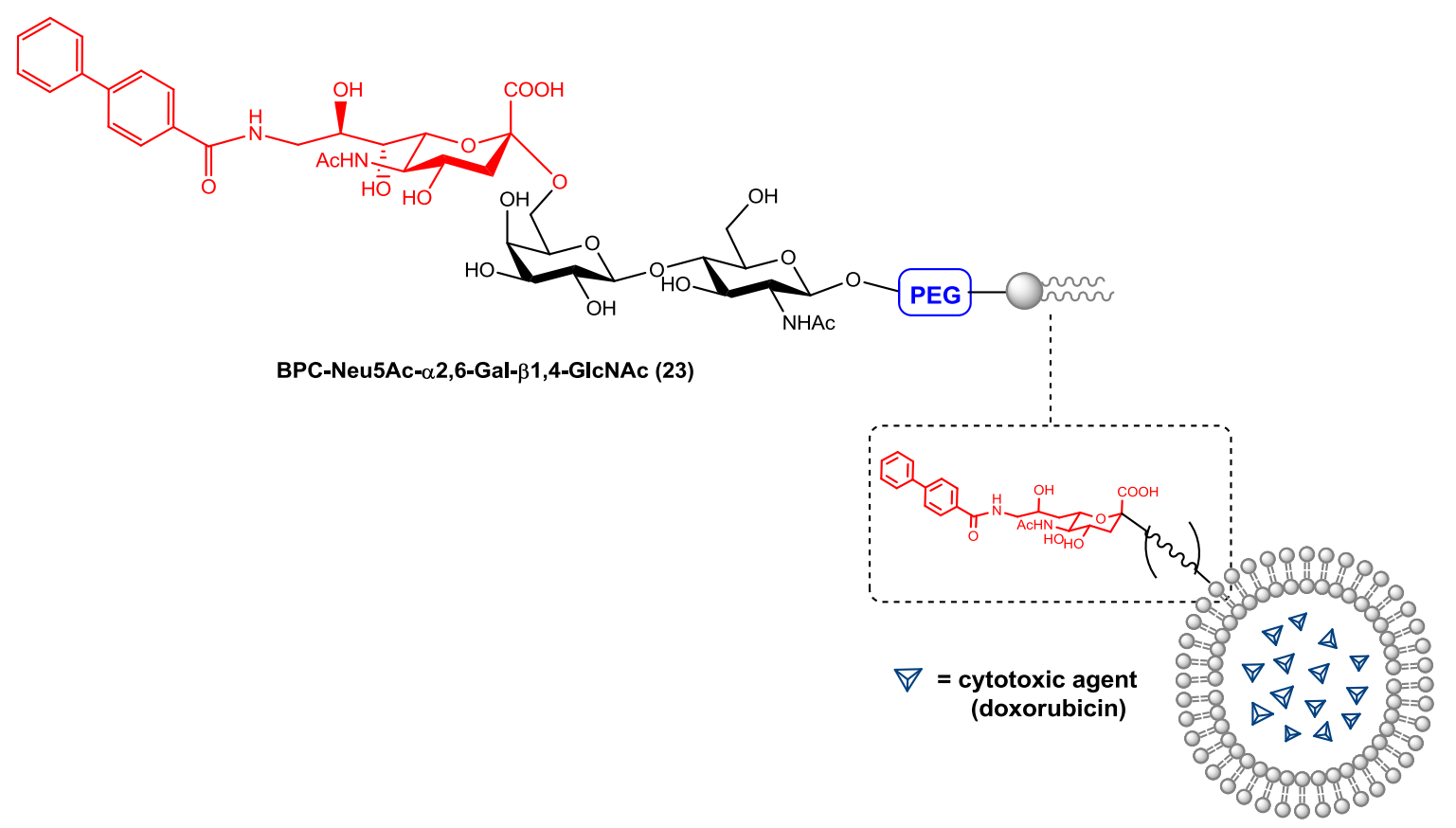

Figure 11. Structure of a liposomal nanoparticle decorated with BPC-Neu5Ac-containing trisaccharide as high-affinity ligand for the lectin CD22 to selectively target B cell lymphoma.

The resulting glycan-displaying nanoparticles selectively target CD22-expressing cells, including B cell lymphoma. By an in vitro viability assay, it was demonstrated that the targeted delivery of the cytotoxic cargo with the nanoparticle strategy was effective in depleting B lymphoma cells, as the cell viability was reduced to less than $15 \%$, compared to non-targeted, liposomal doxorubicin treatment (cell viability $80 \%$ ). ${ }^{[31]}$ An additional advantage of the above liposomal formulation is that the glycan ligands are displayed in a multivalent fashion, which is beneficial for their interactions with siglecs. A disadvantage of this particular glycan epitope is that the high affinity and selectivity of BPC-Neu5Ac$\alpha 2,6-G a l-\beta 1,4-G I c N A c(23)$ is somewhat derogated by a cross-reactivity of the glycan-ligand with the structurally related lectin sialoadhesin (Siglec-1), which is expressed on macrophages. ${ }^{[30,31]}$ As phagocytic cells, macrophages eliminate the liposomes after recognition, which reduces the 
efficiency of drug delivery to malignant cells. However, this problem can be overcome by the design and use of alternative high-affinity ligands, such as other modified sialic acid derivatives with an improved selectivity. ${ }^{[30,32]}$

Subsequent to this, recent significant advances were reported in the development of alternative selective ligands that target the lectins CD22 and CD33. In order to optimise the affinity and selectivity of the previously identified siglec ligands, further alterations to the sialoside scaffold were made. The introduction of a 4-cyclohexyl-1,2,3-triazole residues at position 5 of sialic acid in combination with meta-substituted moieties at position 9 yielded an improved affinity and selectivity profile. Particularly, 3,5-dimethyl benzamide substituents with an additional hydroxyl group in the ortho-position provided the highest avidity for CD33. This was confirmed by solution phase inhibition assays. The sialoside with alterations at both the positions 5 and 9 of the sialic acid moiety (24) exhibited an $\mathrm{IC}_{50}$ of $11 \mu \mathrm{M}$, compared to the monosubstituted analogue (at position 5) (25), which gave an $\mathrm{IC}_{50}$ of $997 \mu \mathrm{M}$ (relative to the natural sialoside, $\alpha 2,6-\mathrm{Neu} 5 \mathrm{Ac}-\mathrm{Gal}-\mathrm{GlcNAc}$ (Figure 12). ${ }^{[33]}$

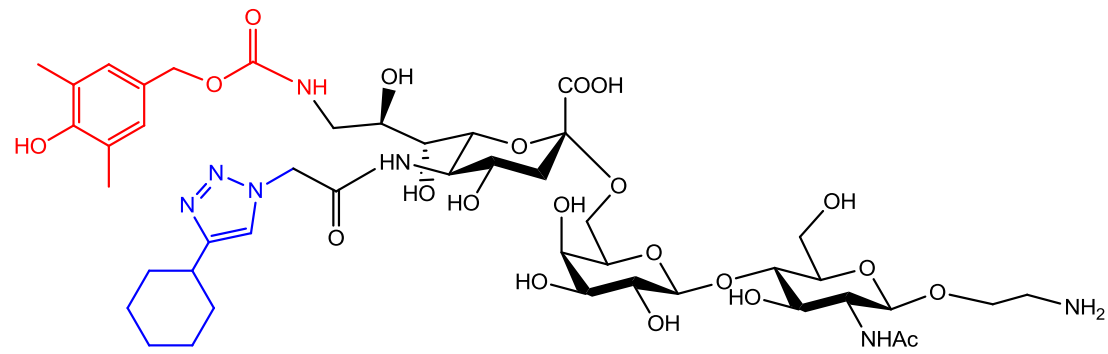

(24)

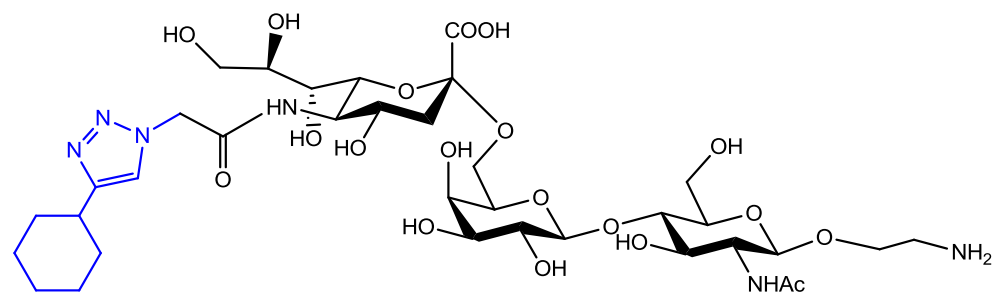

(25)

Figure 12. Development of high-affinity ligands for CD33.

The installation of meta-substituents to the benzamide moiety at position 9 of sialic acid was also exploited for the investigation of more selective sialic acid ligands for Siglec-2 (CD22). In previous studies, it was shown that fluorination of the $\mathrm{N}$-acetamido group at position 5 offers an increased affinity to CD22. Therefore, this feature at position 5 was combined with the meta-substituted benzamide at position 9 and used in the design of CD22 ligands. Indeed, this disubstituted sialoside yielded a very potent and more selective ligand for CD22 (IC 50 of $0,2 \mu \mathrm{M})(\mathbf{2 6})$ (Figure 13). ${ }^{[33]}$ 


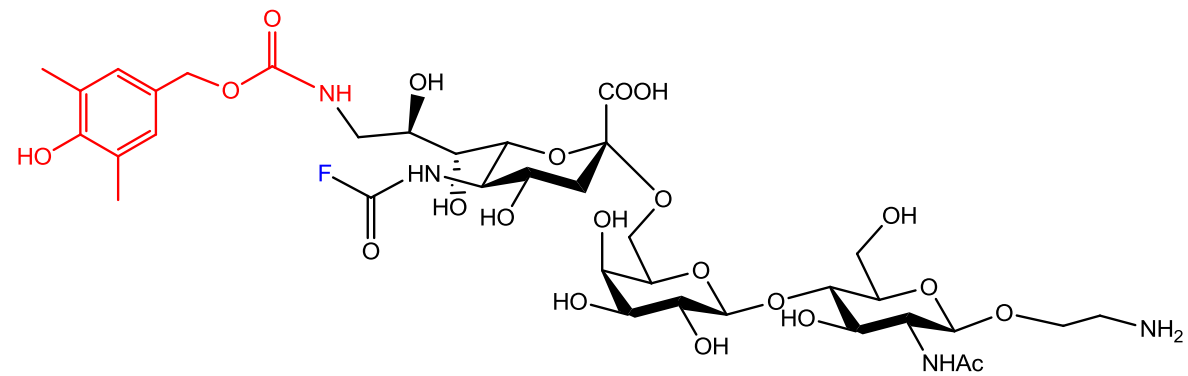

(26)

Figure 13. Development of a high-affinity ligand for CD22.

To assess the therapeutic potential of the newly identified siglec ligands on a cellular level, the sialoside analogues were incorporated into liposomes, as described above, followed by their incubation with CD33-expressing AML cell lines and a CD22-expressing non-Hodgkin's lymphoma Bcell line. These cell studies confirmed the sialosides' high affinity and selectivity for CD33 and CD22 respectively, as the glycan-displaying nanoparticles bound exclusively to their respective target siglec in a ligand-dependent manner. ${ }^{[33]}$

The next challenge is to apply these compounds to in vivo cancer models and investigate whether the nanoparticle strategy proves efficient in delivering cytotoxic compounds to CD33-expressing cells in the same way as it was demonstrated for the CD22-targeted drug delivery approach as exemplified above. ${ }^{[33]}$

\section{Immunotherapeutic approaches}

\section{Therapeutic antibodies}

Therapeutic antibodies represent an alternative cell-directed approach for the treatment of

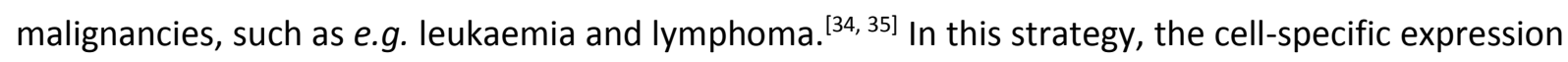
of siglecs on haematopoietic cells, e.g. CD33 on leukaemia cells and CD22 on B-lymphoma cells, is exploited. Monoclonal antibodies (mab), e.g. anti-siglec-antibodies are conjugated to toxins or chemotherapeutic agents, which deliver the cytotoxic moiety upon contact with the antigen. Examples of therapeutic antibodies are gemtuzumab (Mylotarg), an anti-CD33-antibody for the treatment of acute myeloid leukaemia (AML), which was approved by the FDA in 2000 and withdrawn from the market in 2010 due to safety concerns ${ }^{[36]}$ and epratuzumab, an anti-CD22antibody for the treatment of non-Hodgkin lymphoma (NHL) and the autoimmune disorder systemic lupus erythematosus (SLE), which is currently under investigation in clinical trials. ${ }^{[35]}$ 


\section{Carbohydrate-based vaccines}

Another area of immunology, in which sialic acids are involved in drug discovery, is the development of carbohydrate-based vaccines against cancer. ${ }^{[5]}$ In the past two decades, there has been considerable interest in the development of immunotherapeutic strategies, which allow the eradication of tumour cells after treatment with conventional methods, such as surgery, radiation and chemotherapy, in order to increase the overall survival by preventing tumour recurrence and metastasis. ${ }^{[37]}$

The rationale for the development of carbohydrate-based vaccines is based on alterations in cell surface glycan composition of tumour cells compared to healthy cells. Aberrant glycosylation patterns containing tumour-associated glycoforms (TACA) can be exploited as targets to direct a vaccine stimulated immune response towards tumour cells in order to induce their elimination. The design and preparation of carbohydrate vaccines is challenging and requires the consideration of certain key aspects to ensure success in clinical applications. As self-antigens, carbohydrates are poorly immunogenic. In order to evoke an immune response, the carbohydrates need to be conjugated to a carrier protein, e.g. keyhole limpet hemocyanin $(\mathrm{KLH})$, to increase their antigenicity. Further, the carrier proteins must provide attachment sites for carbohydrate antigen conjugation. In addition, the heterogeneous and complex presentation of glycan-antigens on the cell surface needs to be taken into account. Vaccine constructs that closely mimic the actual tumour cell surface glycan are required to activate $T$ cells and elicit a strong antibody production, specific to the carbohydrate epitopes of tumour cells. ${ }^{[38,39]}$ Chemical synthesis methods have immensely contributed to the construction of carbohydrate vaccines. They were applied to the synthesis of carbohydrate-antigen components, peptide backbones, linkers and the conjugation of each component to accomplish the vaccine construct.

Diverse avenues were pursued, including a first generation of single component vaccines, which contain a single carbohydrate antigen attached to an immunogenic carrier (monomeric vaccine) or a single type of carbohydrate antigen in multiple display (clustered monomeric vaccine) as well as more elaborate, multicomponent vaccine constructs (unimolecular multivalent vaccines), which display several different carbohydrate epitopes on a single peptide backbone. ${ }^{[38]}$

Theratope, a sTn-KLH conjugate, represents a carbohydrate-based monomeric vaccine that entered clinical practice in 2002 for the indications metastatic breast cancer and colorectal cancer. Despite initial encouraging results obtained from phase II clinical trials with respect to overall survival rates, results that confirm a significant increase of the survival time could not be reproduced in phase III clinical trials in 2003. ${ }^{[40]}$ 
A representative of a multicomponent vaccine candidate, targeting prostate and ovarian cancer, was designed by Danishefsky and associates. This unimolecular, pentavalent vaccine construct (Figure 14) is composed of five different TACA, namely Globo $\mathrm{H}$, sTn, Tn, TF and Le ${ }^{y}$ antigens attached to a MUC1 glycopeptide backbone conjugated to $\mathrm{KLH} \cdot{ }^{[37]}$

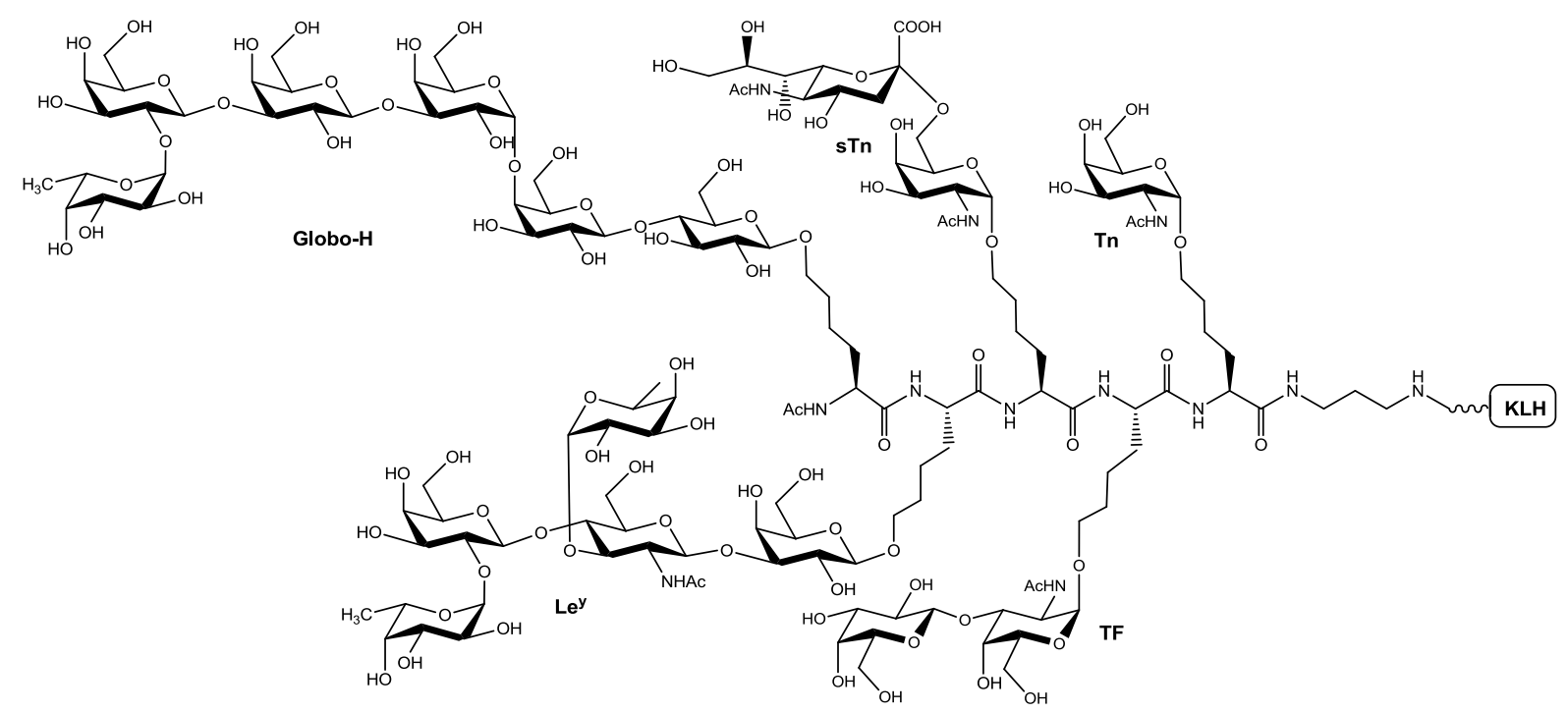

Figure 14. Structure of a unimolecular, pentavalent vaccine construct.

The display of five structurally different TACA on a peptide intends to mimic the tumour cell's natural carbohydrate epitope composition, which could potentially result in a more effective and varied immune response specific to the antigen pattern of a particular cancer type. ${ }^{[38]}$ Indeed, preclinical studies indicated that antibodies were raised to all of the TACA except Le $\mathrm{L}^{\mathrm{y}}$. With the replacement of the Le ${ }^{y}-T A C A$ with the GM2 antigen, the immunogenicity was effectively enhanced, yielding a promising vaccine candidate that entered phase I clinical trials. ${ }^{[41]}$

An impressive example that underlines the achievements of modern drug discovery is illustrated by the development of the Globo $\mathrm{H}$ cancer vaccine. The initial idea of the design of anticancer vaccines arose more than 20 years ago. ${ }^{[42]}$ Since then, continuous efforts have been made in developing a synthetic route that gives access to the Globo $\mathrm{H}$ hexasaccharide conjugated to the KLH carrier protein. A number of different approaches, including first- and second generation syntheses as well as more cost effective programmable one-pot syntheses were established. Further, the introduction of a large-scale enzymatic synthesis method was an important milestone in the development of the Globo $\mathrm{H}$ vaccine. ${ }^{[42]}$ Phase I clinical studies with the anticancer vaccine produced encouraging results so that the candidate was advanced to further clinical development. One of the advantages of using Globo $\mathrm{H}$ as the carbohydrate epitope for the design of vaccines is that the expression of Globo $\mathrm{H}$ glycans is unique to cancer cells and not found on healthy cells. In addition, a number of epithelial cancers, including breast cancers, were shown to be Globo H-positive. Not only Globo H, but also 
other glycans of the Globo series, such as the sialic acid containing SSEA3 (Gb5) and SSEA4 were detected on breast cancer cells and other types of cancer. ${ }^{[42]}$ The occurrence of these epitopes on cancer cells suggest the potentially broad applicability, which vaccines with antigens of the Globo $\mathrm{H}$ series might have in the anticancer field.

The role of glycosphingolipids of the Globo series in cancer, their biosynthesis as well as the interactions with their receptors remain to be identified in the future, revealing perhaps other potential targets, such as the enzymes involved in their biosynthesis or binding proteins, for novel therapeutic strategies. The route of development of this promising new vaccine candidate exemplifies the strength of the interdisciplinary approach, combining synthetic chemistry, cancer biology and immunology, to contribute significant advances to the treatment of cancer.

\section{Stabilisation of biopharmaceutics}

Apart from their roles as targets in drug discovery, sialic acids also serve as stabilising components for biopharmaceutical preparations. In the same way as pegylation is used to reduce the proteolytic cleavage of biopharmaceutical proteins, such as tumour necrosis factor $\alpha$ (TNF $\alpha$ ), interferons (IFN), or asparaginase, sialylation is exploited to improve the pharmacokinetic properties of biotherapeutics by increasing their serum half-lives. Examples where hypersialylation has been employed to confer an enhanced stability to the biopharmaceutical preparation are the hormones luteinising hormone (LH) and erythropoietin (EPO). ${ }^{[43]}$

\section{Conclusions and future perspectives}

In summary, the above examples of therapeutic strategies give insights into the wide implications of sialic acids in chemical biology and drug discovery. Examples of sialic acid based drugs that have entered clinical practice as well as potential promising candidates for future disease intervention schemes, with particular consideration of cancer and immunological diseases, have been discussed. Although particular attention has been given to sialic acids in cancer and immunological diseases, it should be noted that the causes of these conditions can be multifaceted and a variety of interacting effector mechanisms are involved in the establishment and progression of such diseases. Likewise well-established pharmaceutical formulations exist that effectively target other carbohydrateindependent processes, such as e.g. cell division or DNA synthesis. Novel sialic acid based drugs may offer alternative therapeutic options and may represent attractive components of combination therapy schemes.

With the clear demonstration that sialic acid containing carbohydrates are valuable targets for chemical biology and medicinal chemistry programmes, recent advances in chemical and enzymatic methods for their preparation are likely to be developed and optimised further, affording entry to a 
wider range of specific isomers of natural and unnatural carbohydrates of interest. These targets will then provide novel biological tools for further deciphering the roles of sialic acids in biological and therapeutic pathways, and could lead to the development of targeted strategies for diseases that we are currently unable to treat in a selective and efficient manner.

\section{Acknowledgements}

The authors would like to thank the European Commission's Seventh Framework Programme FP7/2007-2013 under grant agreement $n^{\circ} 215536$ for funding the Marie Curie Initial Training Network EuroGlycoArrays.

\section{Financial and competing interest disclosure}

The authors have no relevant affiliations or financial involvement with any organisations or entity with a financial interest in or financial conflict with the subject matter or materials discussed in the manuscript.

\section{Executive summary}

- From a chemical perspective, sialic acids differ from other carbohydrates in a number of ways and hence present a number of synthetic challenges.

- Sialic acids influence the structure and function of glycoconjugates and, as ligands for lectins, antibodies and enzymes, they mediate essential cell interaction processes in both healthy and diseased states. As such they play important roles in cell communication and information transfer.

- In cancer or immunological diseases, the sialylation profile of cells in affected tissues is altered. Ultimately, this is a result of changes in the expression level of enzymes.

- Cell surface sialylation correlates with natural killer (NK) cell cytotoxicity. Hypersialylation is exploited by the tumour cell to evade recognition by NK cells, thus protecting the tumour from attack by the immune system.

- The identification of a selective high-affinity ligand for Siglec-7 adds a new perspective to anticancer therapy and is a valuable finding for the elucidation of Siglec utility and function.

- Carbohydrate-derived drug candidates present challenges, particularly with respect to their pharmacokinetic profiles. Their bioavailability is poor. They have a short plasma half-life, a poor metabolic stability and are rapidly excreted.

- The development of a liposomal nanoparticle strategy that selectively targets CD22-expressing cells for the treatment of $B$ cell lymphoma was a milestone in modern drug discovery. 
- Promising immunotherapeutic strategies, including carbohydrate-based vaccines, were developed to promote the eradication of tumour cells after treatment with conventional methods, such as surgery, radiation and chemotherapy, in order to increase the overall survival by preventing tumour recurrence and metastasis.

- Sialic acids have wide implications in chemical biology and drug discovery. Novel sialic acid based drugs may offer alternative therapeutic options and may present attractive components of combination therapy schemes.

\section{Bibliography}

1. Marth JD, Grewal PK. Mammalian glycosylation in immunity. Nature Reviews Immunology 8(11), 874-887 (2008).

2. Ohtsubo K, Marth JD. Glycosylation in cellular mechanisms of health and disease. Cell 126(5), 855-867 (2006).

3. Dube DH, Bertozzi CR. Glycans in cancer and inflammation. Potential for therapeutics and diagnostics. Nature Reviews Drug Discovery 4(6), 477-488 (2005).

4. Fuster MM, Esko JD. The sweet and sour of cancer: Glycans as novel therapeutic targets. Nature Reviews Cancer 5, 526-542 (2005).

5. Galan MC, Benito-Alifonso D, Watt GM. Carbohydrate chemistry in drug discovery. Organic \& Biomolecular Chemistry 9(10), 3598-3610 (2011).

6. Bernardes GJL, Castagner B, Seeberger PH. Combined approaches to the synthesis and study of glycoproteins. ACS Chemical Biology 4(9), 703-713 (2009).

7. Chen X, Varki A. Advances in the biology and chemistry of sialic acids. ACS Chemical Biology 5(2), 163-176 (2010).

8. Schauer R. Achievements and challenges of sialic acid research. Glycoconjugate Journal 17(79), 485-499 (2000).

9. Schauer R. Sialic acids: Chemistry, metabolism and function. Springer Verlag, (1982).

10. Varki A. Sialic acids in human health and disease. Trends in Molecular Medicine 14(8), 351360 (2008).

11. Hudak JE, Canham SM, Bertozzi CR. Glycocalyx engineering reveals a siglec-based mechanism for nk cell immunoevasion. Nature Chemical Biology 10, 69-77 (2014).

${ }^{* *}$ A comprehensive study on the role of sialosides on Siglec function and NK cell cytotoxicity.

12. Chokhawala HA, Huang SS, Lau K et al. Combinatorial chemoenzymatic synthesis and highthrough put screening of sialosides. Acs Chemical Biology 3(9), 567-576 (2008).

13. Crocker PR, Varki A. Siglecs in the immune system. Immunology 103(2), 137-145 (2001).

14. Stevens J, Blixt O, Paulson JC, Wilson IA. Glycan microarray technologies: Tools to survey host specificity of influenza viruses. Nature Reviews Microbiology 4(11), 857-864 (2006). 
15. Varki A, Cummings, R.D., Esko, J.D., Freeze, H.H., Stanley, P., Bertozzi, C.R., Hart, G.W., Etzler, M.W. Essentials of glycobiology. (2). Cold Spring Harbour Press, (2008).

16. Rao FV, Rich JR, Rakic B et al. Structural insight into mammalian sialyltransferases. Nature Structural \& Molecular Biology 16(11), 1186-1188 (2009).

17. Rillahan C, Schwartz E, Rademacher C et al. On-chip synthesis and screening of a sialoside library yields a high affinity ligand for siglec-7. ACS Chemical Biology 8, 1417-1422 (2013).

18. Macauley MS, Crocker PR, Paulson JC. Siglec-mediated regulation of immune cell function in disease. Nature Reviews Immunology 14, 653-666 (2014).

19. Cazet A, Julien S, Bobowski M, Burchell J, Delannoy P. Tumour-associated carbohydrate antigens in breast cancer. Breast Cancer Research 12(3), (2010).

20. Cazet $A$, Julien S, Bobowski $M$ et al. Consequences of the expression of sialylated antigens in breast cancer. Carbohydrate Research 345(10), 1377-1383 (2010).

21. Ernst B, Magnani JL. From carbohydrate leads to glycomimetic drugs. Nature Reviews Drug Discovery 8(8), 661-677 (2009).

22. Lowe JB, Marth JD. A genetic approach to mammalian glycan function. Annu. Rev. Biochem. 72, 643-691 (2003).

23. Filser C, Kowalczyk D, Jones $C$ et al. Synthetic glycopeptides from the e-selectin ligand 1 with varied sialyl lewis(x) structure as cell-adhesion inhibitors of e-selectin. Angewandte ChemieInternational Edition 46(12), 2108-2111 (2007).

24. Anonymous. Deal watch: Pfizer deal for selectin inhibitor highlights potential of glycomimetic drugs. Nature reviews. Drug discovery 10(12), 890-890 (2011).

25. http://www.clinicaltrials.gov/.

26. Watz $\mathrm{H}$, Bock $\mathrm{D}$, Meyer $\mathrm{M}$ et al. Inhaled pan-selectin antagonist bimosiamose attenuates airway inflammation in copd. Pulmonary pharmacology \& therapeutics 26(2), 265-270 (2013).

27. Mesch S, Moser D, Strasser DS et al. Low molecular weight antagonists of the myelinassociated glycoprotein: Synthesis, docking, and biological evaluation. Journal of Medicinal Chemistry 53(4), 1597-1615 (2010).

28. Magano J. Synthetic approaches to the neuraminidase inhibitors zanamivir (Relenza) and oseltamivir phosphate (Tamiflu) for the treatment of influenza. Chemical Reviews 109(9), 4398-4438 (2009).

29. Pera NP, Branderhorst HM, Kooij R et al. Rapid screening of lectins for multivalency effects with a glycodendrimer microarray. Chembiochem 11(13), 1896-1904 (2010).

30. Chen WC, Sigal DS, Saven A, Paulson JC. Targeting b lymphoma with nanoparticles bearing glycan ligands of cd22. Leukemia \& Lymphoma 53(2), 208-210 (2012). 
31. Chen WC, Kawasaki N, Nycholat CM et al. Antigen delivery to macrophages using liposomal nanoparticles targeting sialoadhesin/cd169. Plos One 7(6), (2012).

32. Blixt O, Han SF, Liao L et al. Sialoside analogue arrays for rapid identification of high affinity siglec ligands. Journal of the American Chemical Society 130(21), 6680-6681 (2008).

33. Rillahan CD, Macauley MS, Schwartz E et al. Disubstituted sialic acid ligands targeting siglecs CD33 and CD22 associated with myeloid leukaemias and b cell lymphomas. Chemical Science 5, 2398-2406 (2014).

** Reveals novel stratgies for cell directed therapies.

34. Jandus C, Simon H-U, Von Gunten S. Targeting siglecs-a novel pharmacological strategy for immuno- and glycotherapy. Biochemical Pharmacology 82(4), 323-332 (2011).

35. O'Reilly MK, Paulson JC. Siglecs as targets for therapy in immune-cell-mediated disease. Trends in Pharmacological Sciences 30(5), 240-248 (2009).

36. http://www.fda.gov/.

37. Zhu J, Wan Q, Ragupathi G, George CM, Livingston PO, Danishefsky SJ. Biologics through chemistry: Total synthesis of a proposed dual-acting vaccine targeting ovarian cancer orchestration of oligosaccharide and polypeptide domains. Journal of the American Chemical Society 131(11), 4151-4158 (2009).

38. Zhu J, Warren JD, Danishefsky SJ. Synthetic carbohydrate-based anticancer vaccines: The memorial sloan-kettering experience. Expert Review of Vaccines 8(10), 1399-1413 (2009).

39. Astronomo RD, Burton DR. Carbohydrate vaccines: Developing sweet solutions to sticky situations? Nature Reviews Drug Discovery 9(4), 308-324 (2010).

40. Zeichner SB. The failed theratope vaccine: 10 years later. The Journal of the American Osteopathic Association 112(8), 482-483 (2012).

41. Wilson RM, Danishefsky SJ. A vision for vaccines built from fully synthetic tumor-associated antigens: From the laboratory to the clinic. Journal of the American Chemical Society 135, 14462-14472 (2013).

42. Danishefsky SJ, Shue Y-K, Chang C-H. Development of Globo-H cancer vaccine. Accounts of Chemical Research 48, 643-652 (2015).

${ }^{* *}$ An impressive example of the path of development of an anticancer vaccine.

43. Byrne B, Donohoe GG, O'Kennedy R. Sialic acids: Carbohydrate moieties that influence the biological and physical properties of biopharmaceutical proteins and living cells. Drug Discovery Today 12(7-8), 319-326 (2007).

\section{Defined key terms:}

\section{Siglecs (page 5)}


Siglecs (sialic acid binding, immunoglobulin-like lectins) are a type of lectins that belong to the immunoglobulin superfamily. They are expressed on cells of the immune and haematopoietic system, including B- and T-cells, NK-cells, dendritic cells, granulocytes as well as mast cells and macrophages.

\section{Selectins (page 5)}

Selectins belong to the class of C-type lectins. A characteristic of this class of lectins is that they have an absolute requirement for $\mathrm{Ca}^{2+}$-ions in order to bind glycans.

\section{NK cells (page 6/7)}

Natural killer cells (NK cells) are effectors of the innate immune system. They respond to infected or stressed cells without the presence of antibodies or major histocompatibility complexes (MHC), which making a fast immune reaction possible.

\section{CuAAC (page 7):}

The $\mathrm{Cu}(\mathrm{I})$-catalysed azide-alkyne cycloaddition belongs to the click-chemistry type of reactions. It is a reliable and practical synthetic strategy that ligates azide and alkyne-functionalised components together upon triazole formation.

\section{CD22 (page 15/16)}

CD22 (Siglec-2) is exclusively expressed on B-cells and is responsible for the regulation of the B-cell dependent immune response, i. e. the inhibition of B-cell activation and B-cell receptor signalling and the homing of B-cells to the bone marrow. It shows a particularly high sensitivity for $\alpha 2,6$-linked sialosides.

\section{CD33 (page 15/16)}

CD33 (Siglec-3) related siglecs are expressed on cells of the haematopoietic system of both lymphoid and myeloid lineages. As inhibitory receptors they contribute to the regulation of the immune response. They recognise $\alpha 2,3$ - and $\alpha 2,6$-sialylated structures.

\section{Globo series (page 19)}

The Globo series of glycans (globosides) comprises a class of glycosphingolipids that feature Gala14Gal linkages. They are found on human erythrocytes and play important roles in recognition and cell differentiation. 


\section{SSEA3 and SSEA4 (page 19)}

Stage-specific embryonic antigen (SSEA)-3 and -4 are a type of cell surface glycosphingolipids that belong to the Globo series of glycans. 